\title{
Domain-Wall Depinning Assisted by Pure Spin Currents
}

\author{
D. Ilgaz, ${ }^{1}$ J. Nievendick, ${ }^{1}$ L. Heyne, ${ }^{1}$ D. Backes, ${ }^{1,2}$ J. Rhensius, ${ }^{1,2}$ T. A. Moore, ${ }^{1, *}$ M. Á. Niño, ${ }^{3}$ A. Locatelli, ${ }^{3}$ \\ T. O. Menteş, ${ }^{3}$ A. v. Schmidsfeld, ${ }^{1}$ A. v. Bieren, ${ }^{1}$ S. Krzyk, ${ }^{1}$ L. J. Heyderman, ${ }^{2}$ and M. Kläui ${ }^{1, \dagger}$ \\ ${ }^{1}$ Fachbereich Physik, Universität Konstanz, Universitätsstraße 10, 78457 Konstanz, Germany \\ ${ }^{2}$ Laboratory for Micro- and Nanotechnology, Paul Scherrer Institut, 5232 Villigen PSI, Switzerland \\ ${ }^{3}$ Sincrotrone Trieste S.C.p.A., Strada Statale 14, 34149 Basovizza, Trieste, Italy
}

(Received 8 December 2009; published 12 August 2010)

\begin{abstract}
We study the depinning of domain walls by pure diffusive spin currents in a nonlocal spin valve structure based on two ferromagnetic Permalloy elements with copper as the nonmagnetic spin conduit. The injected spin current is absorbed by the second Permalloy structure with a domain wall, and from the dependence of the wall depinning field on the spin current density we find an efficiency of $6 \times$ $10^{-14} \mathrm{~T} /\left(\mathrm{A} / \mathrm{m}^{2}\right)$, which is more than an order of magnitude larger than for conventional current induced domain-wall motion. Theoretically we find that this high efficiency arises from the surface torques exerted by the absorbed spin current that lead to efficient depinning.
\end{abstract}

Spin currents and magnetoresistance effects have received much attention over the past two decades [1]. The reciprocal influence of spin-polarized charge currents on the magnetization, which leads to switching in multilayer pillars [2] and the manipulation of magnetic domain walls (DWs) by currents, has become the focus of research due to the fundamental physics as well as possible applications $[3,4]$. The manipulation of DWs has been attempted using spin-polarized charge currents [5-7] or local Oersted fields [8]. However, DW depinning using Oersted fields is facing challenges regarding the scalability, and for spin-polarized charge currents, the effective nonadiabatic torque is small for Permalloy with wide walls $(\beta \ll 1)$ [9-13]. Furthermore, charge currents lead to Joule heating, and at increased temperatures the spin torque efficiency further decreases [14].

A possible alternative approach is to employ pure spin currents, where the electrons diffuse without an associated net charge current. While the generation of spin currents involves energy dissipation, it can occur at a distant location from the device, which can thus be kept cool and still manipulated by the absorbed diffusive spin currents.

Nonlocal spin valves (NLSVs) are promising geometries to generate pure spin currents across transparent [15-17] or tunneling contacts [18,19]. Recently, Yang et al. have demonstrated the reversal of the magnetic state of a Permalloy disk in a NLSV geometry, where the device could be used either in a nonlocal or a lateral spin valve contact setup, and the same critical current densities were observed in both cases since the same torques are involved [20]. This is expected to be radically different for the case of a domain wall in a ferromagnetic wire, where the adiabatic and nonadiabatic torques exerted by a combined charge and spin-polarized current flowing in the wire across the DW and the torques exerted by a spin current absorbed at the DW position will be fundamentally different, but to date this has not been investigated.
In this Letter we present depinning of DWs assisted by pure spin currents. We determine the spin diffusion length in $\mathrm{Cu}$ and the spin polarization in Permalloy $\left(\mathrm{Ni}_{80} \mathrm{Fe}_{20}\right.$, $\mathrm{Py}$ ), and from this we calculate the spin current that diffuses into the ferromagnetic structure where a DW is positioned. We measure the spin current assisted DW depinning and find a large efficiency of the spin current induced torques. This can be explained by the large interface torque that stems from the perpendicular orientation of the magnetization in the domain wall with respect to the spins in the spin current acting on the surface layers.

The two samples examined here (referred to as $A$ and $B$ hereafter) were fabricated in a two-step lithography process [21], and a scanning electron microscope image of such a sample is shown in Fig. 1(a). In Fig. 1(b), an x-ray magnetic circular dichroism photoemission electron microscopy (XMCD PEEM) [22] image of the magnetization configuration with a DW and a corresponding micromag-

(a)

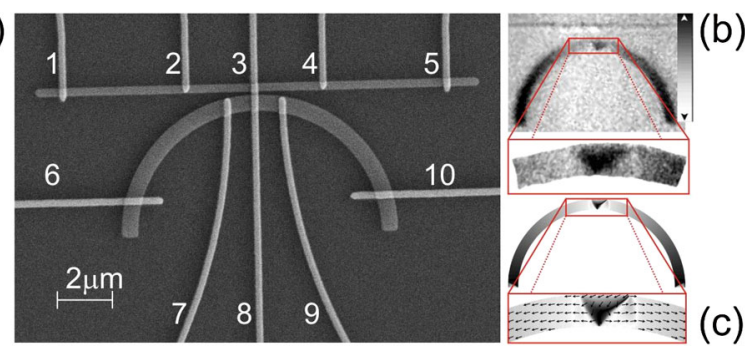

FIG. 1 (color online). (a) Scanning electron microscopy image of the nonlocal spin valve geometry of the samples used in the experiment with the contacts numbered 1-10. The bright stripes are the $\mathrm{Cu}$ contacts, while the darker stripes are the Py wire and half-ring. (b) XMCD PEEM image of the spin configuration and the enlarged image of the transverse domain wall prior to contacting. The shades of gray correspond to the vertical component of the magnetization configurations, which is in agreement with the micromagnetic simulation shown in (c). 
netic simulation [23] using the same geometry are presented [Fig. 1(c)]. First, the two Py elements shown in Fig. 1(a) were deposited with a thickness of $17 \mathrm{~nm}$ and a width of 300 and $600 \mathrm{~nm}$ for the wire and the half-ring wire, respectively. Before the deposition of $50 \mathrm{~nm}$ copper $(\mathrm{Cu})$ as the nonmagnetic material, ion milling was used to clean the interface. On top of the Cu layers, $2 \mathrm{~nm} \mathrm{Au}$ was deposited to prevent oxidation. The width of the central $\mathrm{Cu}$ wire is $330 \mathrm{~nm}$ for sample $A$ and $490 \mathrm{~nm}$ for sample $B$. The edge-to-edge distance between the Py wires was $295 \mathrm{~nm}$ in sample $A$ and $110 \mathrm{~nm}$ in sample $B$. We did not take advantage of tunnel barriers at the ferromagneticnonferromagnetic interfaces, since, although better injection efficiencies can be achieved [18,19], this strongly limits the maximum charge current. Transport measurements were conducted in a cryostat at $4.2 \mathrm{~K}$ using a standard lock-in technique and an in-plane rotatable external magnetic field.

We measure the nonlocal spin signal on both samples (see Fig. 2) with a peak applied charge current density of $2 \times 10^{11} \mathrm{~A} / \mathrm{m}^{2}$ in the Py wire. The origin of these signals is a charge current $I_{C}$ driven from the ferromagnet (FM) into a nonferromagnet (NM), which generates a spin accumulation diffusing to the second ferromagnet [16]. From the calculations in [24,25], for a nonlocal geometry with two different FMs, one obtains $\Delta V_{\mathrm{NL}}(d)$ [see Eq. (1)], the nonlocal spin voltage at the second interface, which describes the difference between a parallel and an antiparallel magnetic configuration:

$$
\Delta V_{\mathrm{NL}}(d)=\frac{\alpha_{F}^{2} I_{C} R_{S, F 1} R_{S, F 2} R_{S, N}}{\exp \left(\frac{d}{\lambda_{N}}\right)\left[R_{S, N}\left(R_{S, F 1}+R_{S, F 2}\right)+2 R_{S, F 1} R_{S, F 2}\right]+R_{S, N}^{2} \sinh \left(\frac{d}{\lambda_{N}}\right)}
$$

Here, $d$ is the distance between both FMs, $\alpha_{F}$ the spin polarization in the FM, and $R_{S, i}$ is the spin resistance with $R_{S, i}=2 \rho_{i} \lambda_{i} /\left[S\left(1-\alpha_{i}^{2}\right)\right]$, where $\rho_{i}$ is the resistivity, $\lambda_{i}$ the spin diffusion length of the specific material, and $S$ the cross-sectional area. The spin current at the second interface is then given by $I_{S}(d)=\Delta V_{\mathrm{NL}}(d) /\left(\alpha_{F} R_{S, F 2}\right)$, and the measured nonlocal change in spin resistance is defined as $\Delta R_{\mathrm{NL}}=\Delta V_{\mathrm{NL}} / I_{C}$.

The jumps in the nonlocal spin resistance signal (Fig. 2) correspond to the switching of the FM wire and half-ring (see sketches in Fig. 2). The spin signal increases from $20.9 \mu \Omega$ for an edge-to-edge distance of $295 \mathrm{~nm}$ in sample $A$ to $88.8 \mu \Omega$ in sample $B$ with a spacing of $110 \mathrm{~nm}$ between both Py wires. Note that the measured spin resistance signal is not symmetric around $0 \Omega$, and this can originate from an inhomogeneous current distribution [26,27]. Furthermore, the switching fields are not exactly equal in both samples, which is probably due to slight variations in the geometry and edge roughness.

We now describe the approach used to determine the spin diffusion length in the $\mathrm{Cu}$ wire and the spin polarization of the Py stripes. Since Py has a small spin diffusion length $[28,29]$, the cross-section areas for both ferromagnets are defined as the interface cross section (width of the Permalloy structure times the width of the $\mathrm{Cu}$ structure). This results in a cubic dependence on the $\mathrm{Cu}$ width of the numerator and a square dependence of all terms in the denominator in Eq. (1). Multiplying Eq. (1) with the $\mathrm{Cu}$ width makes the right-hand side independent of the $\mathrm{Cu}$ width, thus allowing us to use two samples with different $\mathrm{Cu}$ widths for the analysis. The resistivities used are $25 \mu \Omega \mathrm{cm}$ for Py value and $2.2 \mu \Omega \mathrm{cm}$ for the $\mathrm{Cu}$, respectively. To determine the spin diffusion length in $\mathrm{Cu}$ and the spin polarization of Py, we use a value of $5 \mathrm{~nm}$ for the spin diffusion length in Py as this has been determined independently by two groups $[28,29]$. By fitting these used values to the modified equation, we obtain a spin polarization of $\alpha_{\mathrm{FM}}=43 \pm 1 \%$ and a spin diffusion length of $\lambda=$ $\lambda_{\mathrm{Cu}}=134 \pm 12 \mathrm{~nm}$. While the spin polarization found is in agreement with results of Soulen et al. [30], the spin diffusion length is less than the values obtained by Ji et al. [17] and Jedema et al. [31].

Using these results, we can calculate the spin current that arrives at the Py half-ring compared to the charge current injected between contacts 3 and 4 and we find a ratio of $I_{S} / I_{C}=(1.2 \pm 0.1) \times 10^{-2}$.

We now employ these spin currents to manipulate the magnetization. We study their influence on the depinning behavior of a transverse DW (TDW) in the half-ring in
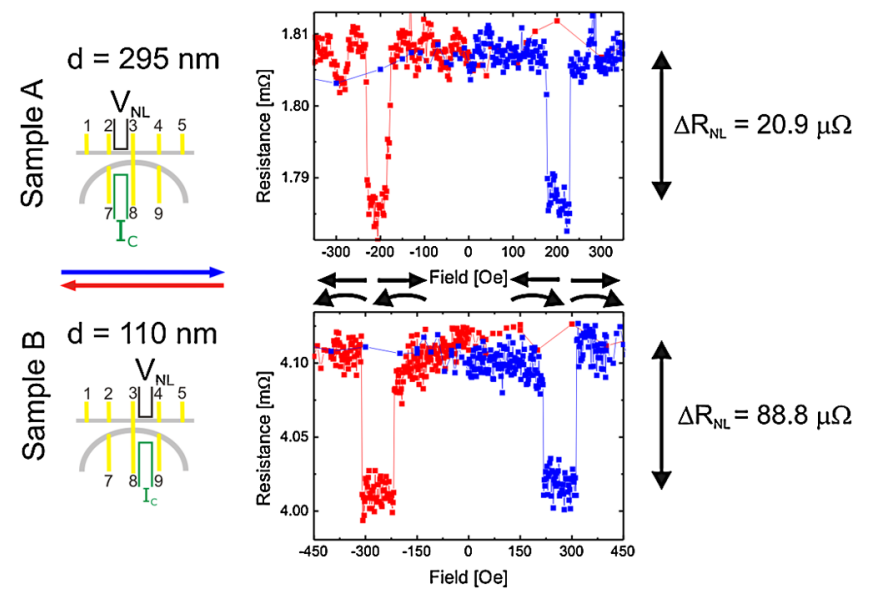

FIG. 2 (color online). Nonlocal spin resistance measurements $\left(R_{\mathrm{NL}} \equiv V_{\mathrm{NL}} / I_{C}\right)$ for both samples with a depiction of the corresponding current and voltage contact setup sketched to the left of the plots and the respective resistance differences to the right. For sample $B$ with a smaller edge-to-edge distance, $d$, between the injector and detector, the nonlocal spin signal increases to $88.8 \mu \Omega$. The arrows between the plots indicate the magnetic orientation of the FM wire and half-ring. 
sample $B$. The TDW was nucleated with a field as described in [32], with a resulting magnetic configuration and a corresponding OOMMF simulation [23] shown in Figs. 1(b) and 1(c). The TDW is positioned below the central $\mathrm{Cu}$ wire slightly off center to the right [see sketch in Fig. 3(d)]. During the experiment a spin current is generated by a $50 \mu$ s long charge pulse between contacts 3 and 4, which is then absorbed by the TDW in the Py halfring. The position and ultimately the depinning of the TDW are determined by the voltage drop due to the anisotropic magnetoresistance signal between contacts 8 and 9 when applying a small ac lock-in current $\left(10^{10} \mathrm{~A} / \mathrm{m}^{2}\right)$ between 3 and 10 (see Fig. 1) [32].
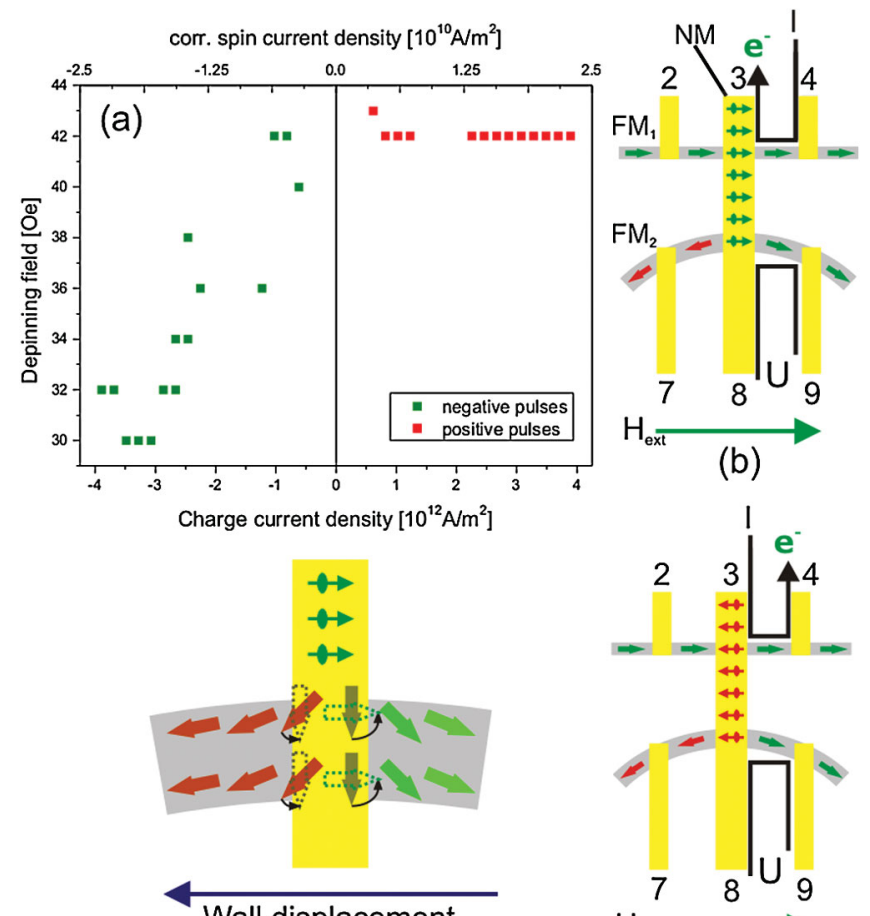

(d)

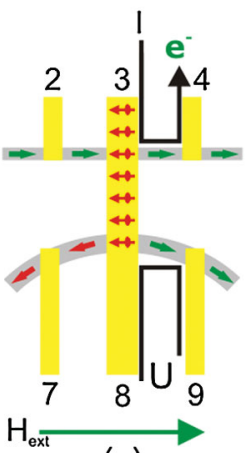

(c)

FIG. 3 (color). (a) Depinning fields as a function of the applied charge currents and the resulting corresponding spin current density (top $x$ axis). The direction of the charge current flow $I$ and the injected pure diffusive spin current (arrows with circles on the central yellow NM spin conduit) are shown in (b) for negative current pulses. The situation for positive pulses is depicted in (c). If the electron (charge) current flows from the ferromagnet $\left(\mathrm{FM}_{1}\right)$ into the nonferromagnet $(\mathrm{NM})$ as in $(\mathrm{b})$, the spins in the spin current (green arrows with circles) are oriented parallel to the magnetization of the ferromagnet $\left(\mathrm{FM}_{1}\right)$. In the opposite case (c) the spins in the spin current (red arrows with circles) are oriented antiparallel to the magnetic orientation of $\mathrm{FM}_{1}$. In (d) we show for negative current pulses the torque [Eq. (2)] exerted by the spin currents that is absorbed from the $\mathrm{NM}$ into the $\mathrm{FM}_{2}$ where the transverse DW is located. The spin current absorption leads to a rotation of the original magnetization (large arrows) in $\mathrm{FM}_{2}$ below the $\mathrm{NM}$ wire counterclockwise [indicated by the black arrows in (d)]. The resulting magnetization direction is shown by the dotted large arrows, meaning that the TDW is effectively displaced to the left.
The dependence of the depinning field as a function of the current amplitude is now shown in Fig. 3(a): For negative currents, the depinning field decreases with increasing current amplitude. For positive currents, where the spin current and the applied field act in opposite directions, one would expect an increase in the depinning field if dc currents are used. But as we use current pulses, the wall depins in between pulses at a field that corresponds to the zero current depinning field, a behavior that we have discussed previously in [14]. The constant depinning field for positive currents also shows that there was no significant Joule heating affecting the depinning. In order to compare our findings with the results of current induced domain-wall motion (CIDM), where the combined spin and charge current flows in the ferromagnet, we divide the spin current by the cross-sectional area of the Py halfring, which results in the spin current densities shown on the upper $x$ axis of Fig. 3(a). We obtain a depinning efficiency $\epsilon=(6 \pm 1) \times 10^{-14} \mathrm{~T} \mathrm{~m}^{2} / \mathrm{A}$ and by linear extrapolation a spin current density of $(7 \pm 2) \times 10^{10} \mathrm{~A} / \mathrm{m}^{2}$ at which the DW would depin without any external field. Compared with CIDM, the efficiency is larger by an order of magnitude $\left(\epsilon_{\mathrm{CIDM}} \approx 5 \times 10^{-15} \mathrm{~T} \mathrm{~m}^{2} / \mathrm{A}\right.$ [14]) and the extrapolated required current density for depinning $\left(j_{\mathrm{CIDM}} \approx 2 \times 10^{12} \mathrm{~A} / \mathrm{m}^{2}\right.$ [14] $)$ is about 30 times smaller.

To demonstrate that it is the spin current that acts on the DW, we simulate the influence of Oersted fields created by the pulses between contacts 3 and 4 with the maximum charge current that was applied in our experiments. We find a maximum field of less than 10 Oe at the edge and an average field of $1 \mathrm{Oe}$ in the area of the DW, which is negligible compared to the depinning field. Furthermore, we have repeated the experiment with the DW at distances of a few hundred $\mathrm{nm}$ from the central $\mathrm{Cu}$ wire, and we see that in this case the depinning of a DW is not affected by the currents, which excludes Oersted field effects and points to depinning via spin current effects.

To theoretically explain the observed high efficiency, we look at the fundamental differences between the torques caused by spin-polarized charge currents flowing in a single Py wire and the lateral spin valve geometry used here. The change of the magnetization $\vec{m}$ (here a dimensionless unit vector) in the case of a spin current being absorbed at a NM-FM interface is given by [33]

$$
\frac{\partial \vec{m}}{\partial t}=\frac{\gamma \hbar}{2 e M_{S} V} \vec{m} \times \vec{I}_{S} \times \vec{m} .
$$

Here, $\hbar$ is the Planck constant, $M_{S}$ the saturation magnetization of the FM, $\gamma=\frac{2 \mu_{B}}{\hbar}$ the gyromagnetic ratio $\left(\mu_{B}\right.$ being the Bohr magneton), $\vec{I}_{S}$ the orientation of the spin current injected into the FM, and $V$ the volume affected by the noncollinear torque. For our case, $V$ is defined by the penetration depth of the spin current multiplied by the surface area where the spin currents enter and act on the FM. This surface area is given by the DW width multiplied by the wire thickness (assuming all the spins enter by the 
side wall) or the wire width plus the thickness (assuming that the spins enter by the side and the top surface of the Py half-ring). In our experiment, the orientation in the spin current $\left(\vec{I}_{S}\right)$ is perpendicular to the magnetization $(\vec{m})$ inside the TDW [see Fig. 3(d)] leading to a maximized torque with a magnitude of

$$
\left|\frac{\partial \vec{m}}{\partial t}\right|_{S C}=\frac{I_{S} \mu_{B}}{e M_{S} V}
$$

This has to be compared with the spin-polarized charge current induced magnetization change. Assuming $\alpha=\beta$ [9-11], the magnitude of the torque for a DW along the $x$ axis is given by [34]

$$
\left|\frac{\partial \vec{m}}{\partial t}\right|_{\mathrm{CIDM}}=\frac{\alpha_{F} I_{C} \mu_{B}}{A e M_{S}}\left|\frac{\partial \vec{m}}{\partial x}\right|
$$

Here, $I_{C}$ is the charge current sent through a FM wire with a cross-sectional area $A$. Dividing Eq. (3) by Eq. (4), we obtain

$$
\frac{|\partial \vec{m} / \partial t|_{S C}}{|\partial \vec{m} / \partial t|_{\mathrm{CIDM}}}=\frac{I_{S} A}{\alpha_{F} I_{C} V(\partial m / \partial x)}
$$

Depending on the volume affected by the spin current, this ratio becomes $\simeq 25$ (assuming the side and the top of the FM) to $\simeq 900$ (assuming only the side). The magnetization gradient $\frac{\partial m}{\partial x}$ is given by the domain-wall width $l_{\mathrm{DW}}: \frac{\partial m}{\partial x}=$ $\frac{2}{l_{\mathrm{DW}}}$ and the magnetic dephasing length used is $0.8 \mathrm{~nm}$ [35]. Thus, these calculations show that for pure spin current induced DW depinning, the impact on the magnetic moments at the edge of the FM wire are 1-2 orders of magnitude larger than for CIDM. For DWs pinned at edge defects, this gives an estimate of the increased depinning efficiency in line with our measurements.

In conclusion, we have shown that the depinning of DWs can be efficiently assisted by nonlocal spin currents due to the large torque that then acts on the surface layers of the FM, where the domain-wall pinning originates.

The authors acknowledge support by the DFG (SFB 767, KL1811), the ERC Starting Independent Researcher Grant (ERC-2007-Stg 208162), the EU RTN Spinswitch (MRTN-CT-2006-035327) and Human Resources and Mobility Programme, as well as the Samsung Advanced Institute of Technology. The research at Elettra has received funding from the European Community's 6th and 7th Framework Programmes ["I3:IA-SFS" and "ELISA" (Contract No. 226716), respectively]. The authors would like to thank A. Brataas for fruitful discussions.

* Now at: School of Physics and Astronomy, University of Leeds, Leeds LS2 9JT, United Kingdom.
${ }^{\dagger}$ Also at: Laboratory of Nanomagnetism and Spin Dynamics, Ecole Polytechnique Fédérale de Lausanne (EPFL), 1015 Lausanne, Switzerland; SwissFEL, Paul Scherrer Institut, 5232 Villigen PSI, Switzerland. mathias@klaeui.de

[1] M. N. Baibich et al., Phys. Rev. Lett. 61, 2472 (1988); G. Binasch et al., Phys. Rev. B 39, 4828 (1989).

[2] J. A. Katine et al., Phys. Rev. Lett. 84, 3149 (2000).

[3] R.P. Cowburn et al., U.K. Patent No. WO/2007/132174, 2007.

[4] S. S. P. Parkin, M. Hayashi, and L. Thomas, Science 320, 190 (2008).

[5] G. Tatara, H. Kohno, and J. Shibata, Phys. Rep. 468, 213 (2008).

[6] A. Yamaguchi et al., Phys. Rev. Lett. 92, 077205 (2004).

[7] M. Kläui et al., Phys. Rev. Lett. 94, 106601 (2005).

[8] D. Ilgaz et al., Appl. Phys. Lett. 93, 132503 (2008).

[9] R. Moriya et al., Nature Phys. 4, 368 (2008).

[10] L. Thomas et al., Nature (London) 443, 197 (2006).

[11] S. Lepadatu et al., Phys. Rev. B 81, 020413 (2010).

[12] T. Moore et al., Phys. Rev. B 80, 132403 (2009).

[13] M. Eltschka et al., Phys. Rev. Lett. 105, 056601 (2010).

[14] M. Laufenberg et al., Phys. Rev. Lett. 97, 046602 (2006).

[15] M. Johnson and R. H. Silsbee, Phys. Rev. Lett. 55, 1790 (1985).

[16] F. J. Jedema, A. T. Filip, and B. J. van Wees, Nature (London) 410, 345 (2001).

[17] Y. Ji et al., Appl. Phys. Lett. 88, 052509 (2006).

[18] S. O. Valenzuela and M. Tinkham, Appl. Phys. Lett. 85, 5914 (2004).

[19] A. Vogel et al., Appl. Phys. Lett. 94, 122510 (2009).

[20] T. Yang, T. Kimura, and Y. Otani, Nature Phys. 4, 851 (2008).

[21] L. J. Heyderman et al., Microelectron. Eng. 73-74, 780 (2004).

[22] J. Stöhr et al., Science 259, 658 (1993).

[23] http://math.nist.gov/oommf/. Cell size, $5 \times 5 \times 17 \mathrm{~nm}$; material, Py $\left(M_{S}=860 \mathrm{kA} / \mathrm{m}, A=1.3 \times 10^{-11} \mathrm{~J} / \mathrm{m}\right)$.

[24] S. Takahashi and S. Maekawa, Phys. Rev. B 67, 052409 (2003); J. Fabian and I. Žutić, in Spintronics-From GMR to Quantum Information, edited by S. Blügel, D. Bürgler, M. Morgenstern, C. M. Schneider, and R. Waser, Lecture Notes of the 40th IFF Spring School (Forschungszentrum Jülich, Jülich, 2009).

[25] T. Kimura, Y. Otani, and J. Hamrle, Phys. Rev. B 73, 132405 (2006).

[26] F. Casanova et al., Phys. Rev. B 79, 184415 (2009).

[27] M. Johnson and R. H. Silsbee, Phys. Rev. B 76, 153107 (2007).

[28] S. Dubois et al., Phys. Rev. B 60, 477 (1999).

[29] S. D. Steenwyk et al., J. Magn. Magn. Mater. 170, L1 (1997).

[30] R. J. Soulen, Jr. et al., Science 282, 85 (1998).

[31] F. J. Jedema et al., Phys. Rev. B 67, 085319 (2003).

[32] M. Kläui et al., Phys. Rev. Lett. 90, 097202 (2003).

[33] Y. Tserkovnyak et al., Rev. Mod. Phys. 77, 1375 (2005).

[34] Z. Li and S. Zhang, Phys. Rev. Lett. 92, 207203 (2004).

[35] S. Urazhdin, R. Loloee, and W. P. Pratt, Jr., Phys. Rev. B 71, 100401 (2005). 